\title{
CCD photometry, spin and shape models of five asteroids: 225, 360, 416,516 , and 1223
}

\author{
T. Michałowski ${ }^{1}$, W. Pych $^{2}$, J. Berthier ${ }^{3}$, A. Kryszczyńska ${ }^{1}$, T. Kwiatkowski ${ }^{1}$, J. Boussuge ${ }^{4}$, S. Fauvaud ${ }^{4}$, \\ P. Denchev ${ }^{5}$, and R. Baranowski ${ }^{1}$ \\ 1 Astronomical Observatory, A. Mickiewicz University, Słoneczna 36, 60-286 Poznań, Poland \\ 2 Astronomical Observatory, Warsaw University, Al. Ujazdowskie 4, 00-478 Warszawa, Poland \\ 3 Institut de Mécanique Céleste, 77 Av. Denfert Rochereau, 75014 Paris, France \\ 4 Astroqueyras Association, 05350 Saint Veran, France \\ ${ }^{5}$ Institute of Astronomy, Rozhen National Observatory, P.O. Box 136, 4700 Smolyan, Bulgaria
}

Received June 9; accepted August 11, 2000

\begin{abstract}
New CCD photometric observations for 225 Henrietta (1995), 360 Carlova (1996, 1997, 1998), 416 Vaticana (1995-96, 1998), 516 Amherstia (1995, 1996), and 1223 Neckar (1995-96) carried out at five observatories are presented. New synodical periods for two asteroids $(225,1223)$ have been obtained. Using all available lightcurves, the spin vectors, senses of rotation, and triaxial elipsoid models of these five asteroids have been determined or refined.
\end{abstract}

Key words: techniques: photometric — minor planets: asteroids

\section{Introduction}

Photometric observations provide the most aboundant data for determinations of rotational periods, orientations of spin vectors, and physical properties of surfaces of asteroids. The photometric database consists of the lightcurves of more than 700 asteroids, but spin vectors have been determined for only about 100 of them. Magnusson et al. (1989) provided a review of all techniques used in order to obtain poles and shapes of asteroids. One approach to obtain this information is to study the brightness variation af asteroids as they spin about their axes. Such a method, described by Michałowski (1993), has been used in the present work.

The present paper is a part of a programme devoted to enlarging the number of asteroids with known physical parameters. The new photometric observations presented here, combined with previously published ones,

Send offprint requests to: T. Michałowski, e-mail: tmich@amu.edu.pl have been used to determine sidereal periods, coordinates of poles and triaxial ellipsoid models for the observed asteroids. These new models will be included into the existing database of spin parameters and used for statistical investigation of the evolution history of the asteroid main belt. The most extensive spin and shape database is the one compiled by Per Magnusson, which can be found in The Small Bodies Node of the NASA Planetary Data System (http://pdssbn.astro.umd.edu/).

\section{Observations}

Photometric observations of five asteroids from 31 nights during the years 1995-1998 were made at five observatories. The majority of data came from Ostrowik (Warsaw University Observatory, Poland) and Pic du Midi (France). During several nights, observations were also carried out at Château Renard (Astroqueyras Association, France), Rozhen (Institute of Astronomy, Bulgaria) and Borowiec (A. Mickiewicz University, Poland).

At Ostrowik, a 60-cm Cassegrain telescope, equipped with a TEK512 CB CCD camera, was used (Udalski \& Pych 1992). CCD frames, collected through the $R$ and $I$ Cousins filters were reduced with standard $I R A F$ procedures and the profile photometry was obtained with the Daophot-II package.

At Pic du Midi, the observing system consists of a 105-cm Cassegrain reflector, a Thomson 7863 CCD camera and an $R$ filter. All reductions and the profile photometry were performed with the ASTROL package, developed at the Institut de Mécanique Céleste in Paris (Kryszczyńska et al. 1996).

On four nights in August 1995, the asteroid 225 Henrietta was also observed at Château Renard 
Observatory in the French Alps. As the asteroid was very bright, all measurements were performed through the $R$ filter with a small 19-cm telescope and a KAF-400 CCD camera. The magnitudes of the asteroid and comparison stars were determinined by aperture photometry after the images were corrected for bias, dark and flat-field.

In March 1997, the asteroid 360 Carlova was observed at two observatories. A 60-cm Cassegrain telescope equipped with a single-channel photometer was used at the Rhozen Observatory (Bulgaria). A transformation to the $U B V$ standard system has been carried out with standard algorithms (Denchev et al. 1998). One lighturve of this object was obtained at Borowiec Station of the Poznan Observatory (Poland), with a 25-cm Newton reflector and a KAF-400 CCD camera. Due to the small aperture of the telescope, no filters were used. Corrections for bias, dark and flat-field were made with CCDOPS program (produced by a camera manufacturing company, SBIG). An aperture photometry was performed with the Daophot II package. After upgrading to the 40-cm Newton reflector, two asteroids (360 and 416) were observed at Borowiec in 1998. This time a Bessel $R$ filter was used. A standard reduction of the CCD frames as well as the aperture photometry were performed with the STARLINK package.

From all the lightcurves, only the Rhozen photoelectric photometry data were transformed to the standard system as has been stated above. The rest of the observations have not been transformed, mainly because of non-photometric weather condition and/or because the observing systems were equipped with only one standard filter.

Table 1 contains the aspect data for the asteroids observed. The first column is the date of the observation referring to the mid-time of the observed lightcurve. The next two columns are the distances (in astronomical units) from the asteroid to the Sun and the Earth, respectively. Column 4 is the solar phase angle, and Cols. 5 and 6 give the J2000.0 ecliptic longitude $(\lambda)$ and latitude $(\beta)$, respectively, referring to the time in the first column. The names of the observatories are listed in the last column of the table.

The results of our observations are presented in Figs. 1-9 as composite lightcurves. They have been obtained with a procedure described in Magnusson \& Lagerkvist (1990). The lightcurves have been composited with the synodical periods shown in the graphs. Points from different nights are marked with different symbols. The vertical position of each individual lightcurve is obtained to minimize the dispersion of data points relative to their neighbours. The abscissae are the rotational phases with the zero points corrected for light-time.

\subsection{Henrietta}

The first photometric observations for this asteroid were reported by Weidenschilling et al. (1990). They observed
Table 1. Aspect data

\begin{tabular}{|c|c|c|c|c|c|c|}
\hline$\overline{\text { Date (UT) }}$ & $(\mathrm{AU})$ & $(\mathrm{AU})$ & $\begin{array}{c}\text { Phase } \\
\text { angle } \\
\left({ }^{\circ}\right)\end{array}$ & $\begin{array}{c}\lambda \\
(\mathrm{J} 2 \\
\left(^{\circ}\right)\end{array}$ & $\begin{array}{c}\beta \\
000) \\
\left({ }^{\circ}\right)\end{array}$ & Obs. \\
\hline \multicolumn{7}{|l|}{225 Henrietta } \\
\hline 19950803.0 & 2.624 & 1.935 & 19.24 & 6.61 & 14.63 & $\mathrm{ChR}$ \\
\hline 19950805.1 & 2.627 & 1.918 & 18.80 & 6.61 & 14.56 & ChR \\
\hline 19950826.0 & 2.667 & 1.775 & 12.74 & 4.91 & 13.46 & ChR \\
\hline 19950827.0 & 2.669 & 1.770 & 12.10 & 4.76 & 13.39 & ChR \\
\hline 19951023.9 & 2.796 & 1.934 & 12.02 & 353.93 & 6.12 & Pic \\
\hline 19951024.7 & 2.798 & 1.941 & 12.25 & 353.86 & 6.02 & Ost \\
\hline 19951025.8 & 2.800 & 1.954 & 12.58 & 353.76 & 5.85 & Ost \\
\hline \multicolumn{7}{|l|}{360 Carlova } \\
\hline 19960119.9 & 2.459 & 1.949 & 21.99 & 49.15 & -13.01 & Pic \\
\hline 19970303.0 & 3.037 & 2.057 & 3.62 & 167.54 & 9.57 & Roz \\
\hline 19970304.0 & 3.039 & 2.058 & 3.45 & 167.32 & 9.61 & Roz \\
\hline 19970311.0 & 3.051 & 2.070 & 3.46 & 165.78 & 9.92 & Bor 25 \\
\hline 19980428.0 & 3.527 & 2.583 & 6.69 & 235.82 & 15.93 & Bor40 \\
\hline 19980501.0 & 3.528 & 2.572 & 6.06 & 235.27 & 15.99 & Bor40 \\
\hline 19980503.0 & 3.528 & 2.565 & 5.67 & 234.88 & 16.02 & Bor40 \\
\hline \multicolumn{7}{|l|}{416 Vaticana } \\
\hline 19951020.1 & 3.056 & 2.076 & 4.01 & 21.24 & -11.23 & Pic \\
\hline 19951022.1 & 3.060 & 2.083 & 4.20 & 20.77 & -11.11 & Pic \\
\hline 19960118.9 & 3.207 & 3.197 & 17.64 & 19.78 & -4.70 & Pic \\
\hline 19980325.8 & 2.681 & 1.827 & 13.39 & 150.54 & 18.55 & Bor 40 \\
\hline 19980326.8 & 2.678 & 1.832 & 13.69 & 150.39 & 18.46 & Bor 40 \\
\hline 19980328.8 & 2.674 & 1.842 & 14.28 & 150.11 & 18.29 & Bor 40 \\
\hline 19980329.8 & 2.671 & 1.847 & 14.58 & 149.97 & 18.20 & Bor 40 \\
\hline \multicolumn{7}{|c|}{516 Amherstia } \\
\hline 19950926.0 & 3.133 & 2.146 & 4.03 & 7.37 & 11.39 & Ost \\
\hline 19951022.9 & 3.183 & 2.281 & 9.01 & 1.56 & 11.92 & Ost \\
\hline 19951023.9 & 3.185 & 2.290 & 9.03 & 1.39 & 11.91 & Ost \\
\hline 19961015.1 & 3.383 & 2.693 & 14.05 & 73.71 & 16.36 & Ost \\
\hline 19961016.1 & 3.382 & 2.682 & 13.88 & 73.64 & 16.42 & Ost \\
\hline 19961111.8 & 3.361 & 2.447 & 7.72 & 69.81 & 17.88 & Ost \\
\hline \multicolumn{7}{|l|}{1223 Neckar } \\
\hline 19951025.1 & 2.699 & 2.088 & 18.93 & 93.43 & 1.81 & Pic \\
\hline 19960115.9 & 2.725 & 1.850 & 11.24 & 81.73 & 2.97 & Ost \\
\hline 19960116.8 & 2.725 & 1.857 & 11.57 & 81.61 & 2.97 & Ost \\
\hline 19960117.7 & 2.725 & 1.865 & 11.88 & 81.50 & 2.96 & Pic \\
\hline
\end{tabular}

Observatory Code: ChR - Château Renard; Pic - Pic du Midi; Ost - Ostrowik; Bor25 - Borowiec, 25 cm; Bor40 - Borowiec, $40 \mathrm{~cm}$.

Henrietta on 22 and 23 May 1982 and determined a synodical period of 8.75 hours from the $0.29 \mathrm{mag}$ amplitude lightcurve. This period gave no overlap between the two nights' data, but yielded equally spaced maxima and minima. Subsequent observations were carried out on 13 Oct. 1983 giving a partial lightcurve (about 4 hours) with an amplitude of $0.16 \mathrm{mag}$.

Zappala et al. (1989) reported their observations from two consecutive nights in March 1987. The lightcurve with an amplitude of 0.15 mag was rather noisy, but according to the authors it fitted both periods of 8.4 and 4.2 hours.

Our observations (see Fig. 1) were performed at three observatories and spanned almost three months. We have obtained a composite lightcurve with a period of 


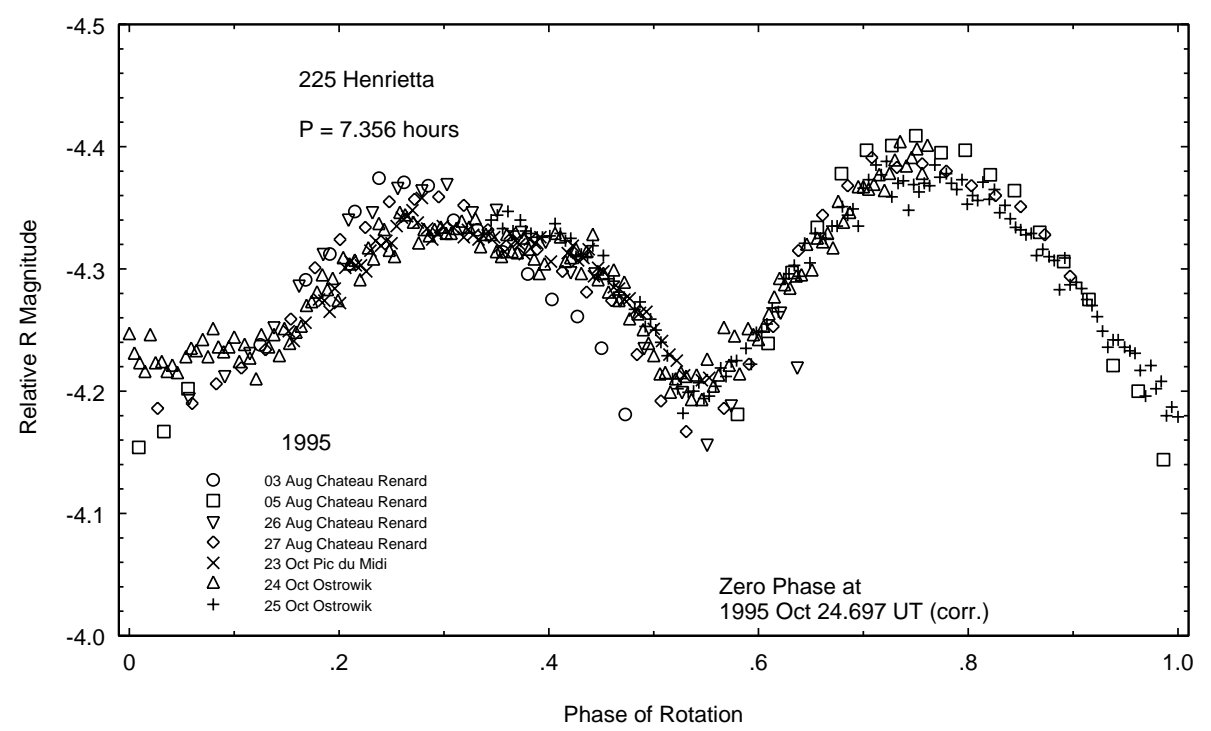

Fig. 1. Lightcurve of 225 Henrietta in 1995

$7.356 \pm 0.001$ hours and an amplitude of 0.22 mag was determined. The notable differencies in the levels of extrema from different nights (see Fig. 1) are due to the effects of phase angle changes on the amplitude of brightness (see Zappala et al. 1990 for details).

The synodical period of 7.356 hours determined by us is shorter than earlier reported values. However, we were not able to obtain any composite lightcurves with the earlier periods. The next step was to check if this shorter period fitted the observations from 1982 and 1987 oppositions. Without any problems we could see that the new period was also sufficient for the lightcurves from the two previous oppositions. So, we can conclude that the synodical period of Henrietta is shorter by about 1 hour than those previously reported.

\subsection{Carlova}

Photometric observations for this asteroid were performed by Harris \& Young (1983) on four consecutive nights in October 1979. These data contained only a few mesurements per night but they allowed the authors to obtain a synodical period of 6.21 hours from the 0.37 mag composite lightcurve. Di Martino et al. (1987) observed Carlova on 21 and 22 September 1984 and reported a rotational period of 6.183 hours. The lightcurve with an amplitude of $0.30 \mathrm{mag}$ was quite irregular and asymmetric with the primary maximum very sharp and narrow compared to the secondary one. On 10 January 1986 this asteroid was observed again by Dotto et al. (1995). They also obtained an asymmetric lightcurve with an amplitude of $0.33 \mathrm{mag}$.

We observed this asteroid on 19 January 1996 but the lightcurve covered only half of the rotational cycle. An amplitude of $0.44 \mathrm{mag}$ was found (Fig. 2). Subsequent data were obtained during three nights in March 1997 (Fig. 3), and a composite lightcurve with an amplitude of 0.30 mag was obtained. The period of $6.188 \pm 0.003$ hours which we obtained is consistent with the previously published values. Carlova was also observed on three nights in AprilMay 1998 (Fig. 4). The lightcurve with an amplitude of $0.49 \mathrm{mag}$, the largest ever observed for this asteroid, confirmed the period of 6.188 hours.

\subsection{Vaticana}

Lagerkvist et al. (1987) obtained three short lightcurves (the longest was about 3 hours) in 1985 but no unique rotation period could be derived. The amplitude of the light variation seemed to be larger than $0.19 \mathrm{mag}$.

There are many observations from the 1989 apparition. Erikson et al. (1991) observed this asteroid on four nights in February while Miles (1990) reported data from eight nights in the period from March - May. A composite lightcurve with an amplitude of 0.4 mag was based on the rotational period of 5.372 hours. Moreover, Miles (1990) determined the phase relation with the parameters: $H=7.90$ and $G=0.21$.

Schober et al. (1994) observed Vaticana on four nights in August 1985. The composite lightcurve, with an amplitude of $0.15 \mathrm{mag}$, confirmed the earlier reported rotational period.

We performed our observations on three nights in October 1995 and January 1996 (Fig. 5). The amplitude was $0.38 \mathrm{mag}$ and the period of 5.372 hours was confirmed. Another lightcurve was obtained during four nights in March 1998. These data confirmed the period of 5.372 hours. The four nights' runs were too short and did not cover the whole rotational cycle (Fig. 6). The lightcurves from March 25 and 29 have been manually 


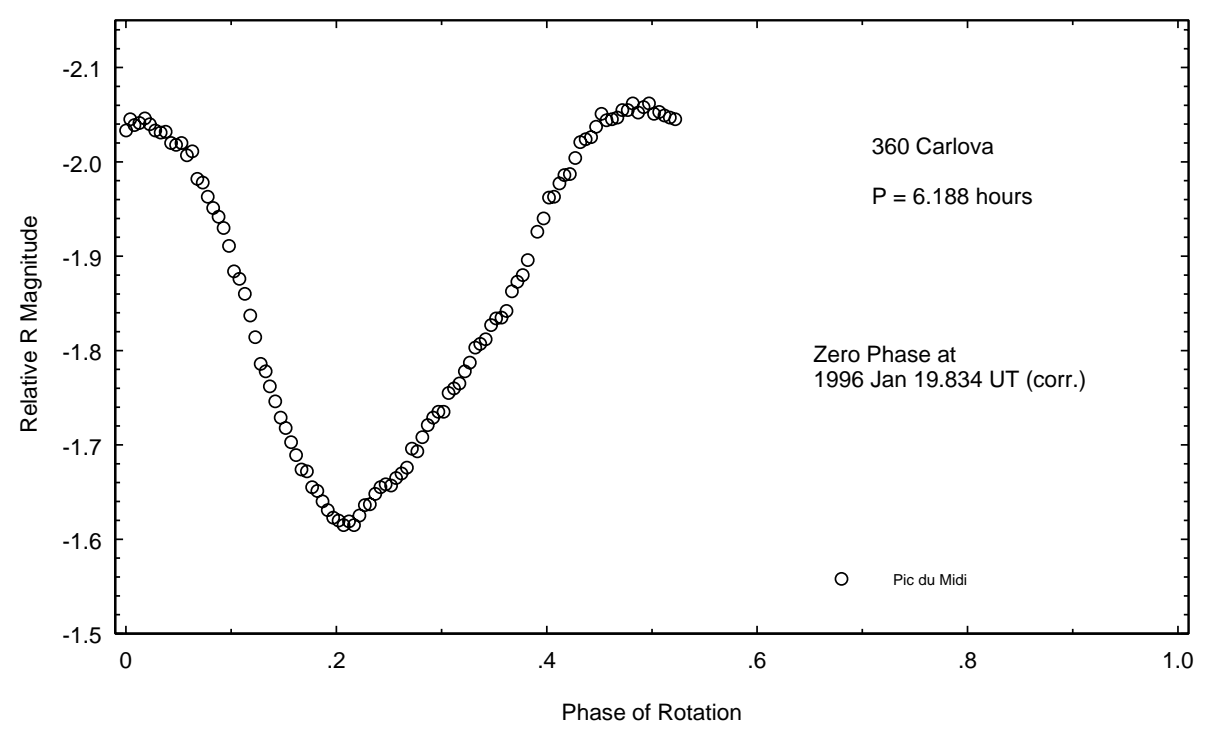

Fig. 2. Lightcurve of 360 Carlova in 1996

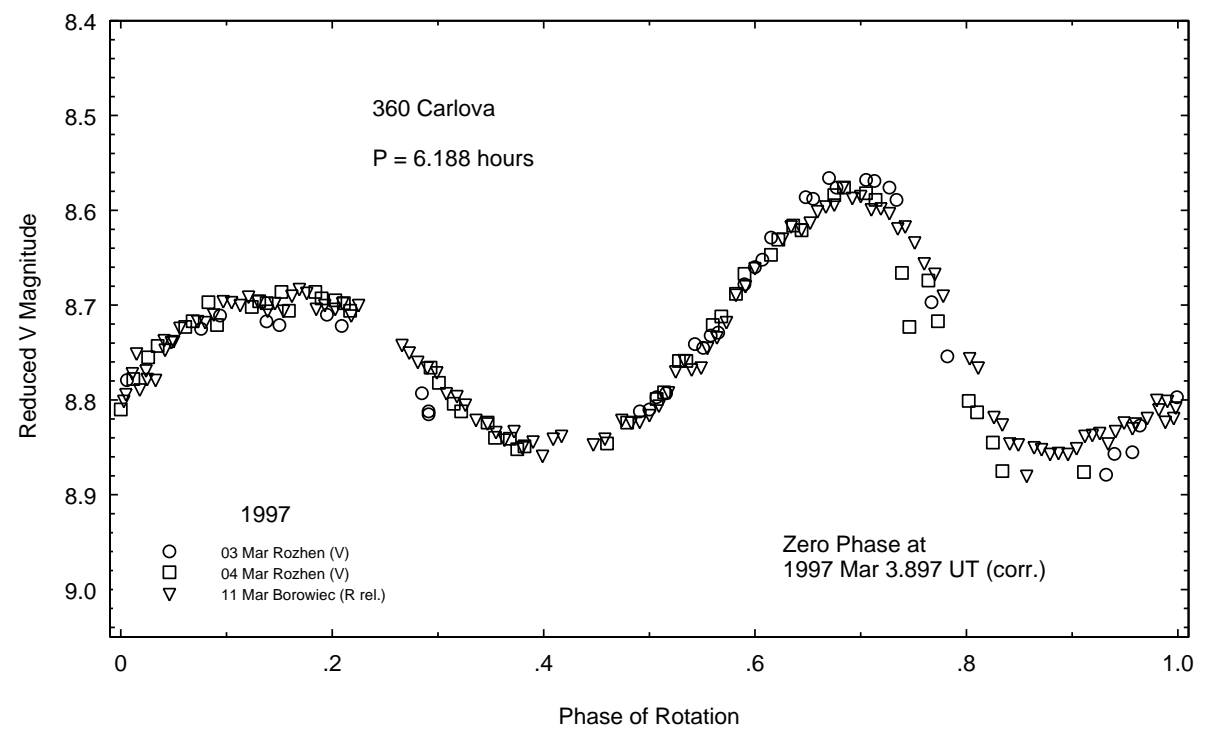

Fig. 3. Lightcurve of 360 Carlova in 1997. The points from 3 Mar are shifted by 0.002 mag in respect to those from 4 Mar

shifted for better display. The amplitude seems to be slightly larger than 0.17 mag.

\subsection{Amherstia}

Harris \& Young (1980) observed this asteroid on 29 October 1978. Fortunately, this data made it possible to obtain an approximate period of $7 \pm 1$ hours, and showed an amplitude of $0.15 \mathrm{mag}$. Other observations were performed by Lagerkvist et al. (1987) on three consecutive nights in March 1985. They derived a period of 7.494 hours and an amplitude of $0.48 \mathrm{mag}$. Amherstia was also observed on 26 January 1989 by Dotto et al. (1992). The lightcurve did not cover the whole rotational cycle but an amplitude of $0.25 \mathrm{mag}$ was visible.
The lightcurves obtained on three nights in September and October 1995 allowed us to obtain a period of $7.484 \pm$ 0.001 hours. The composite lightcurve with an amplitude of $0.41 \mathrm{mag}$ is displayed in Fig. 7. Some discrepancies in the levels of the deeper minimum from different runs are due to differences in the phase angle on different nights. The observations from October - November 1996 confirmed the period of 7.484 hours but the amplitude was smaller - $0.15 \mathrm{mag}$ (Fig. 8).

\subsection{Neckar}

This asteroid was observed by Tedesco (1979) on one night in February 1977. The lightcurve with an amplitude of $0.4 \mathrm{mag}$ and two pairs of extrema covered 7.5 hours. It indicated a period of $8.6 \pm 0.5$ hours. Binzel (1987) observed 


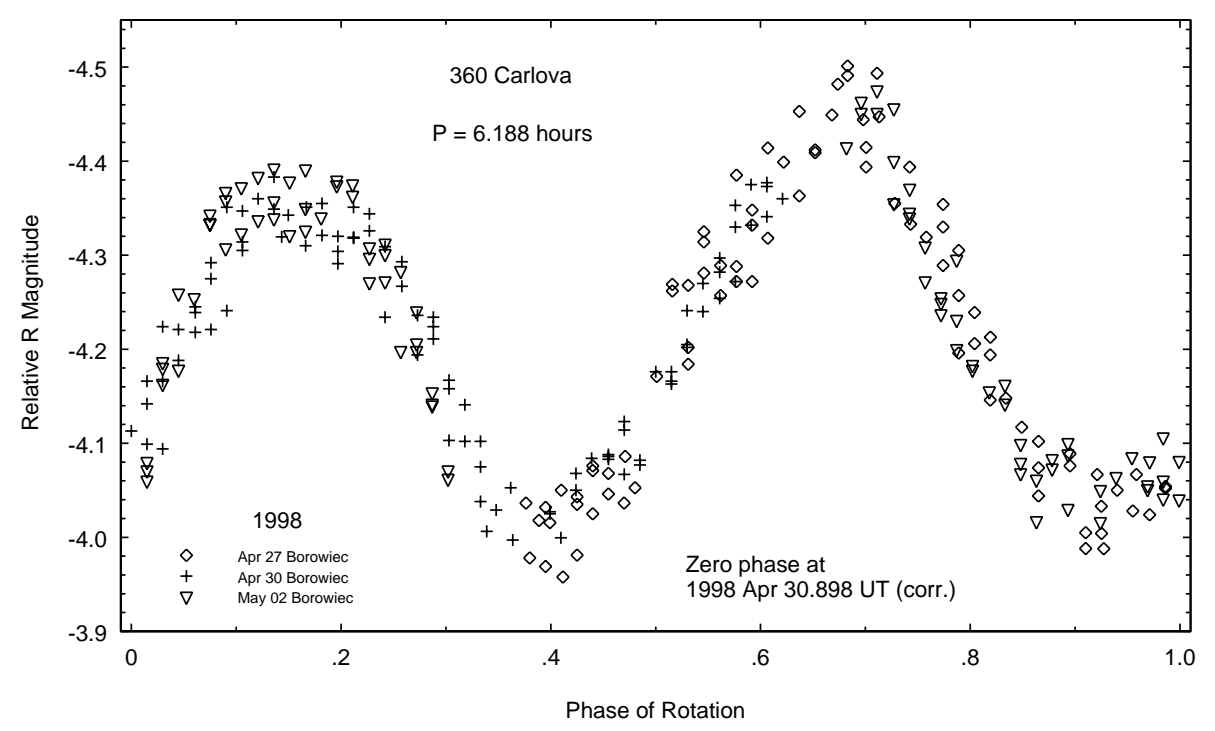

Fig. 4. Lightcurve of 360 Carlova in 1998

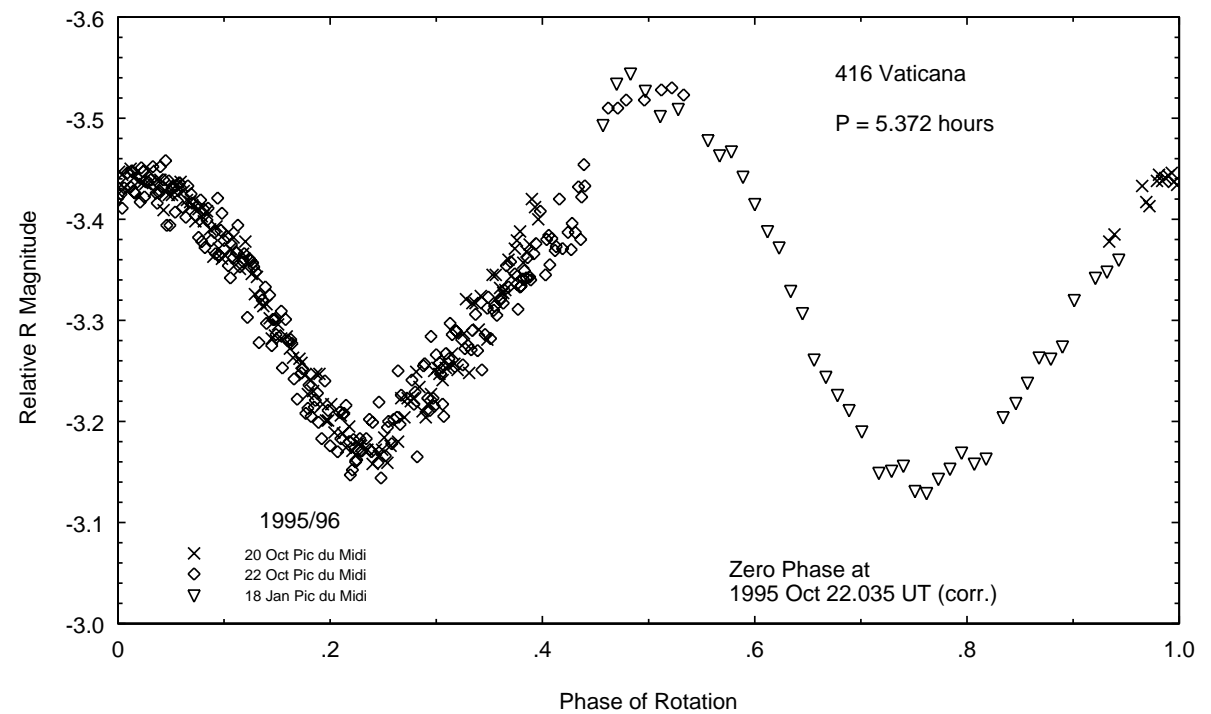

Fig. 5. Lightcurve of 416 Vaticana in 1995-96

Neckar on four consecutive nights in May 1983. The amplitude of $0.14 \mathrm{mag}$ was significantly lower than in 1977 , indicating a more polar aspect. The composite lightcurve was constructed with a rotational period of $8.78 \pm$ 0.02 hours. However, that lightcurve displayed three distinct pairs of extrema per rotational cycle.

Slivan \& Binzel (1996) observed Neckar on two and three consecutive nights during four apparitions: March 1987, September 1989, November 1990 and May 1993. These data led to a new rotational period of $7.81 \pm$ 0.03 hours, but they did not comment on why the previous values were incorrect. The amplitudes of these lightcurves are in the range of $0.16-0.45 \mathrm{mag}$.

We observed Neckar on four nights in the 1995-96 apparition (see Fig. 9) covering almost three months. Our observations do not confirm the previously determined periods, as shown above. We have obtained a composite lightcurve with a period of $7.763 \pm 0.001$ hours. The amplitude of this asymmetric lightcurve, with two maxima at different levels, is 0.18 mag. If we used the period of 7.81 hours determined by Slivan \& Binzel (1996) to composite our observations, than the switch of the maxima between October 1995 and January 1996 observations would be visible. That is why the shorter period of 7.763 hours, determined in the present study, is correct.

The observations by Tedesco (1979), carried out in one night, also confirm the shorter period obtained in the present work. Our observations contain a few hundred points while those by Binzel (1987) only 34 measurements (only a few points per night). It was easy for us to make a new composite lightcurve which also confirmed the shorter period. Moreover, the lightcurve 


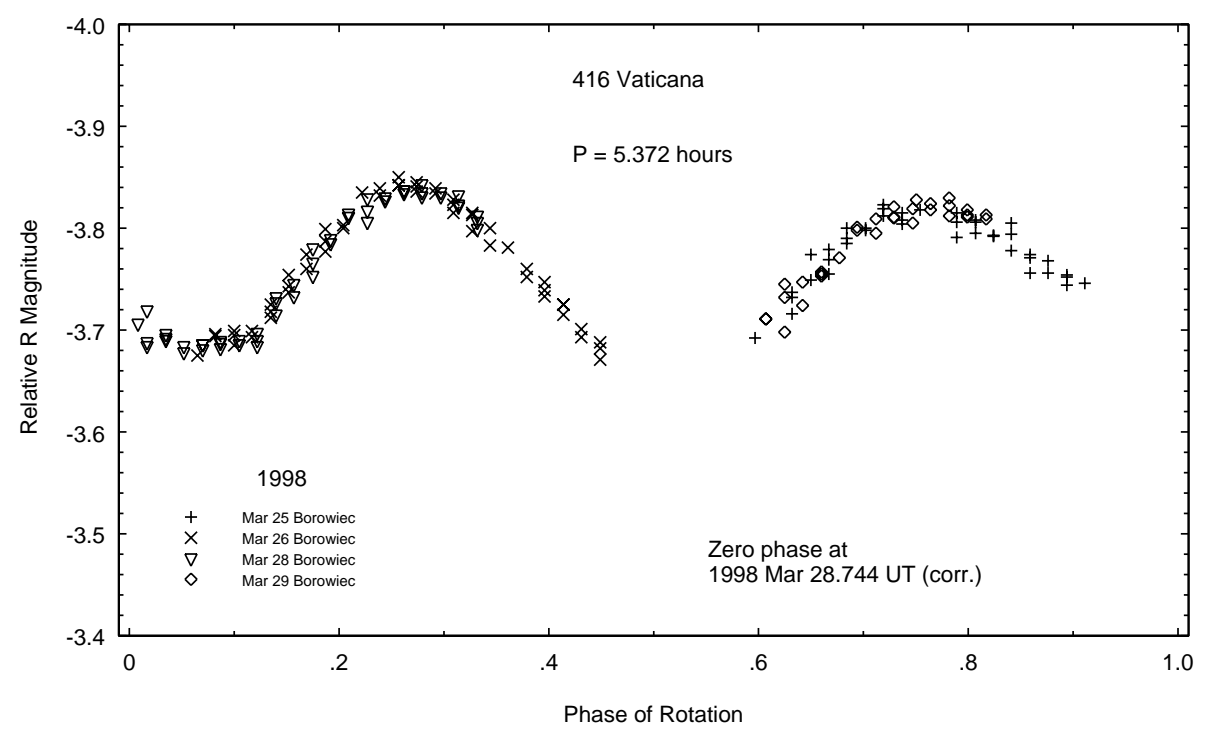

Fig. 6. Lightcurve of 416 Vaticana in 1998. The data from March 25 and 29 are shifted manually for better display

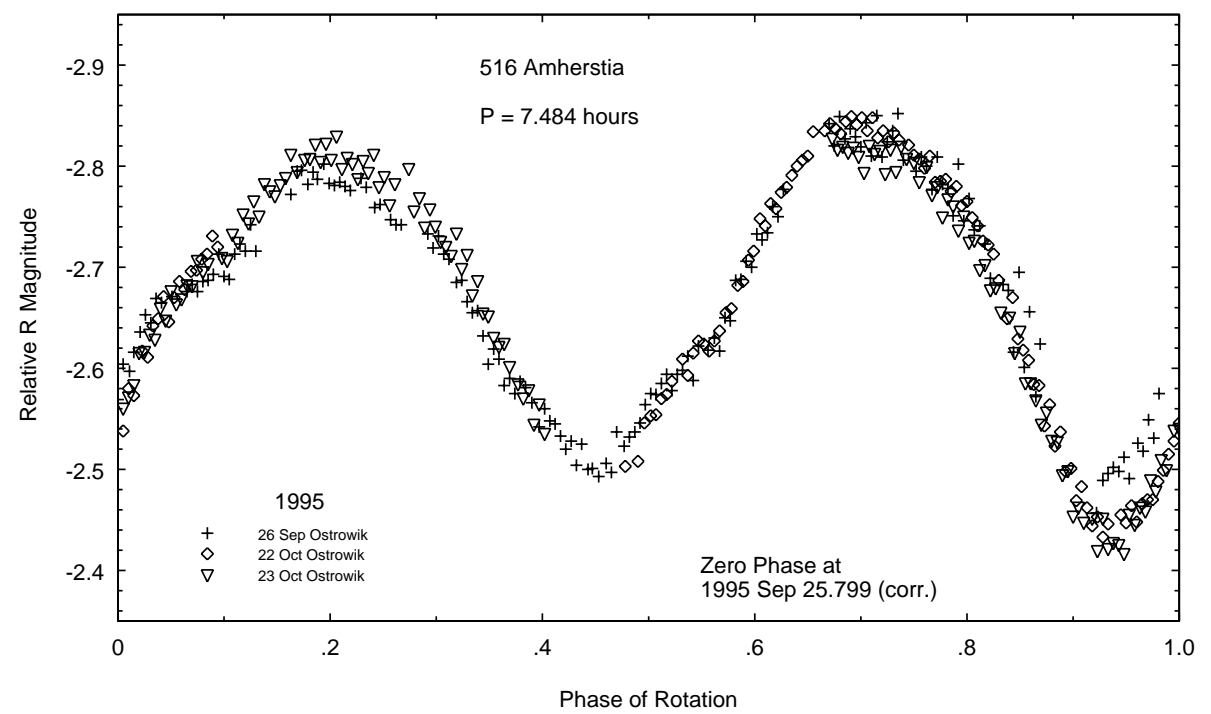

Fig. 7. Lightcurve of 516 Amherstia in 1995

obtained showed two maxima and two minima per rotational period. The lightcurves from Slivan \& Binzel (1996) also confirm the period reported in the present paper. So, we can conclude that the synodical period of 7.763 hours is consistent with all available observations from seven oppositions (1977, 1983, 1987, 1989, 1990, 1993, 1995-96).

\section{Pole and shape of the observed asteroids}

The spin vectors, sidreal periods, and triaxial ellipsoid models for the observed asteroids were determined by the method described by Michałowski (1993). In this method the magnitudes, amplitudes, and epochs of maxima are considered. The results were obtained by building a set of nonlinear equations whose solution was found by least square fitting. The observed amplitudes and magnitudes of the brightness maxima were reduced to zero phase angle by using the amplitude-phase (Zappala et al. 1990) and the $H G$-magnitude system (Bowell et al. 1989), respectively.

As described by Michałowski (1993), the method had two-fold ambiguity in the location of an asteroid pole when the sense of rotation was fixed. This problem was also discussed earlier in a review paper by Magnusson et al. (1989). They stated that by combining the solutions from amplitude-magnitude and epoch methods, it was sometimes possible to obtain a unique solution even though each individual method had failed to achive this. However, they did not discuss when such situations were possible.

The method used in the present paper combines these methods (instead of solutions alone) in one process of 


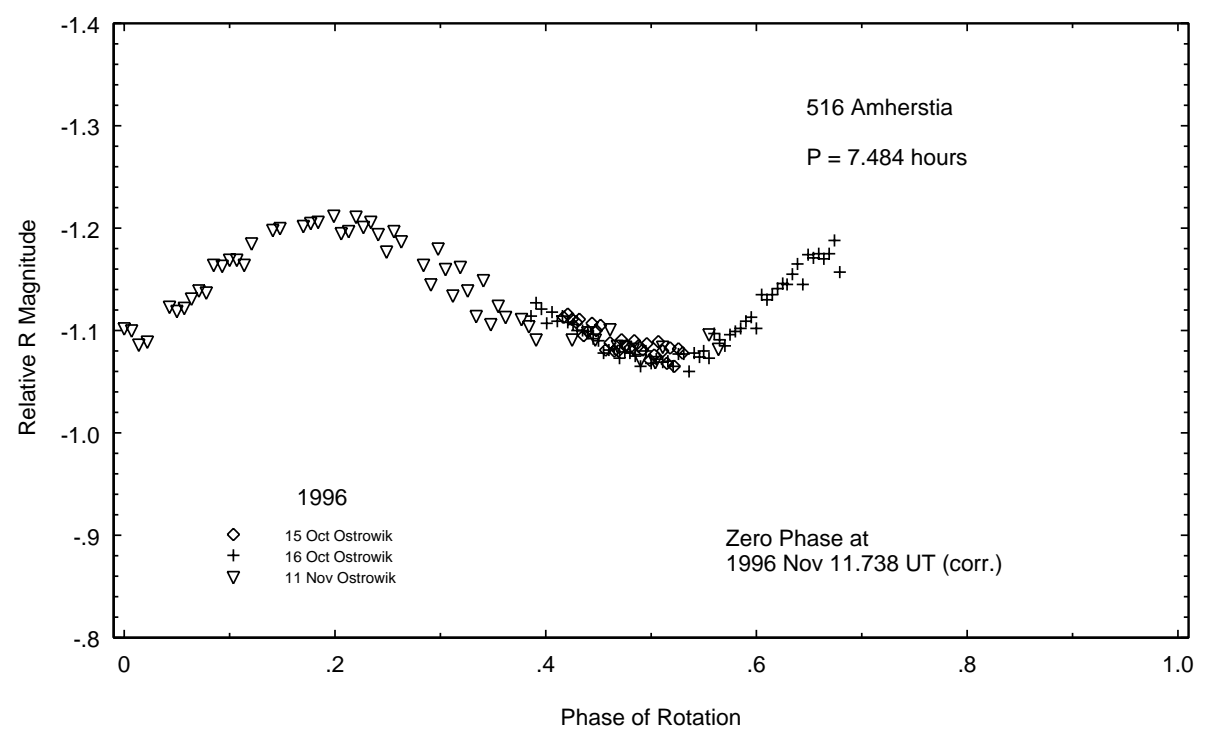

Fig. 8. Lightcurve of 516 Amherstia in 1996

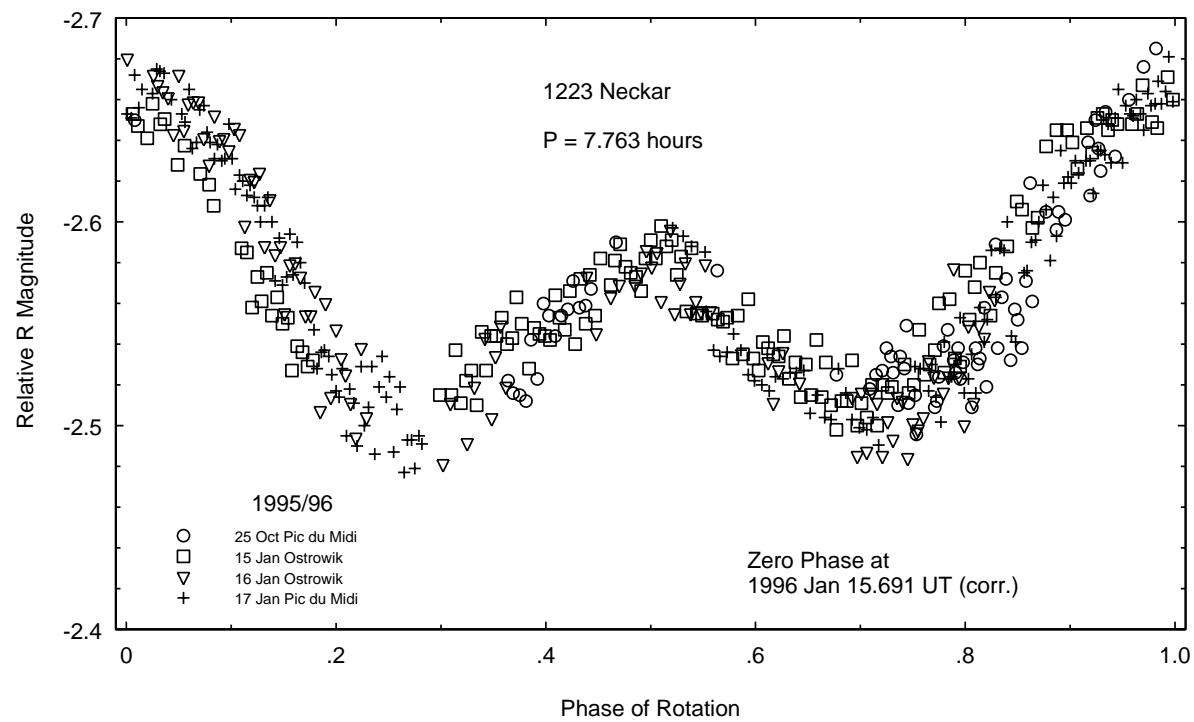

Fig. 9. Lightcurve of 1223 Neckar in 1995-96

Table 2. Asteroid parameters

\begin{tabular}{lcccccc}
\hline Asteroid & $D(\mathrm{~km})$ & albedo & Type & $H(90,0)$ & $G$ & $m$ \\
\hline 225 Henrietta & 120 & 0.040 & $\mathrm{~F}$ & 8.57 & $(0.04)$ & 0.031 \\
360 Carlova & 116 & 0.054 & $\mathrm{C}$ & 8.13 & $(0.04)$ & $(0.010)$ \\
416 Vaticana & 85 & 0.169 & $\mathrm{~S}$ & 7.84 & 0.30 & 0.026 \\
516 Amherstia & 73 & 0.163 & $\mathrm{M}$ & 8.09 & $(0.21)$ & 0.038 \\
1223 Neckar & & & $\mathrm{S}$ & 10.61 & $(0.23)$ & 0.017 \\
\hline
\end{tabular}

calculation as mentioned above. From the results obtained here (see below) and in other papers (Michałowski 1993, 1996; Michałowski et al. 1995; Kryszczyńska et al. 1996) we can also state that in some cases it is possible to obtain a unique solution for the pole location. This possibility can sometimes occur when an asteroid is observed during a few oppositions and the ecliptic latitudes are within a wide range of values (usually from about $-20^{\circ}$ to $+20^{\circ}$ ). An example of such an asteroid in the present work is 360 Carlova (see below). We have also noticed that for an asteroid which has always been observed in low ecliptic latitudes (e.g. 1223 Neckar from the present study), we have obtained two solutions for its pole coordinates and the two-fold ambiguity is not broken. 
Table 3. Spin and shape models

\begin{tabular}{|c|c|c|c|c|c|c|c|c|c|}
\hline \multirow{2}{*}{$\begin{array}{r}\text { Sidereal } \\
\text { period (days) }\end{array}$} & \multirow{2}{*}{$\begin{array}{l}\text { Sense of } \\
\text { rotation }\end{array}$} & \multicolumn{2}{|c|}{ Pole 1} & \multicolumn{2}{|c|}{ Pole 2} & \multirow[t]{2}{*}{$a / b$} & \multirow[t]{2}{*}{$b / c$} & \multirow[t]{2}{*}{ Method } & \multirow[t]{2}{*}{ Reference } \\
\hline & & $\lambda_{\mathrm{p}}$ & $\beta_{\mathrm{p}}$ & $\lambda_{\mathrm{p}}$ & $\beta_{\mathrm{p}}$ & & & & \\
\hline \multicolumn{10}{|l|}{225 Henrietta } \\
\hline & & $135^{\circ}$ & $13^{\circ}$ & & & 1.23 & 1.08 & EAM & Present work \\
\hline & & $\pm 4^{\circ}$ & $\pm 6^{\circ}$ & & & \pm 0.03 & \pm 0.05 & & \\
\hline \multicolumn{10}{|l|}{360 Carlova } \\
\hline & & $108^{\circ}$ & $+51^{\circ}$ & $337^{\circ}$ & $+47^{\circ}$ & 1.57 & 1.00 & $\mathrm{EA}$ & Dotto et al. (1995) \\
\hline 0.2578997 & $\mathrm{P}$ & $105^{\circ}$ & $+47^{\circ}$ & & & 1.42 & 1.52 & EAM & Present work \\
\hline \pm 0.0000001 & & $\pm 9^{\circ}$ & $\pm 10^{\circ}$ & & & \pm 0.02 & \pm 0.11 & & \\
\hline \multicolumn{10}{|l|}{416 Vaticana } \\
\hline 0.2238486 & $\mathrm{P}$ & $132^{\circ}$ & $+58^{\circ}$ & & & 1.55 & 1.20 & EAM & Present work \\
\hline \pm 0.0000001 & & $\pm 4^{\circ}$ & $\pm 3^{\circ}$ & & & \pm 0.01 & \pm 0.02 & & \\
\hline 0.2238486 & $\mathrm{P}$ & & & $310^{\circ}$ & $+22^{\circ}$ & 1.45 & 1.17 & EAM & Present work \\
\hline \pm 0.0000002 & & & & $\pm 7^{\circ}$ & $\pm 6^{\circ}$ & \pm 0.02 & \pm 0.06 & & \\
\hline \multicolumn{10}{|l|}{516 Amherstia } \\
\hline & & $75^{\circ}$ & $+63^{\circ}$ & $256^{\circ}$ & $+55^{\circ}$ & 1.83 & 1.85 & EA & De Angelis (1995) \\
\hline & & $76^{\circ}$ & $+30^{\circ}$ & & & 1.53 & 1.23 & EAM & Michałowski (1996) \\
\hline 0.3116334 & $\mathrm{P}$ & $75^{\circ}$ & $+17^{\circ}$ & & & 1.36 & 1.82 & EAM & Present work \\
\hline \pm 0.0000003 & & $\pm 5^{\circ}$ & $\pm 3^{\circ}$ & & & \pm 0.01 & \pm 0.04 & & \\
\hline 0.3116332 & $\mathrm{R}$ & $255^{\circ}$ & $-15^{\circ}$ & & & 1.36 & 1.81 & EAM & Present work \\
\hline \pm 0.0000003 & & $\pm 5^{\circ}$ & $\pm 4^{\circ}$ & & & \pm 0.01 & \pm 0.04 & & \\
\hline \multicolumn{10}{|l|}{1223 Neckar } \\
\hline 0.3232105 & $\mathrm{P}$ & $70^{\circ}$ & $+45^{\circ}$ & $255^{\circ}$ & $+42^{\circ}$ & 1.47 & 1.28 & EAM & Present work \\
\hline \pm 0.0000004 & & $\pm 8^{\circ}$ & $\pm 6^{\circ}$ & $\pm 7^{\circ}$ & $\pm 6^{\circ}$ & \pm 0.04 & \pm 0.05 & & \\
\hline
\end{tabular}

The basic parameters of the asteroids are summarized in Table 2. Their IRAS diameters $(D)$ and albedos are taken from The Small Bodies Node of the NASA Planetary Data System (http://pdssbn.astro.umd.edu/), while the taxonomic types are from Tholen (1989). The maximum brightness of the asteroid obtained for aspect $90^{\circ}$ and zero solar angle $H(90,0)$ is shown in the next column. The last two columns display the $G$ and $m$ values obtained during reduction the magnitudes and amplitudes to zero phase angle, respectively. If the existing data were insufficient for such reduction, the average value for a given taxonomic type was taken: from Harris (1989) for $G$ parameter and from Zappala et al. (1990) for $m$. The assumed values are given in parentheses. This table is not complete as the asteroid 1223 Neckar was not observed by the IRAS satellite.

Table 3 contains the spin and shape models for the asteroids studied in the present paper. This table shows the sidereal periods, the senses of rotation ( $\mathrm{P}$ - prograde, $\mathrm{R}$ - retrograde), the ecliptic coordinates (equinox 2000) of the north poles, and the ratios $a / b, b / c$ of triaxial ellipsoid models. The available results obtained by other authors are given for comparison. The methods, used for calculation, are also given ( $\mathrm{E}$ - Epochs, A - Amplitude,
M - Magnitude). If no previous results are listed in Table 3, it means the results from the present work are the first published ones for a given asteroid.

\subsection{Henrietta}

No model has been previously reported for this asteroid. We have used the data from four oppositions: 1982, 1983, 1987, and 1995. During these apparitions the ecliptic latitude of Henrietta ranged from $-11^{\circ}$ to $+29^{\circ}$. The results are presented in Table 3 . The available data are insufficient for obtaining a unique solution for the sidereal period and sense of rotation. New observations are needed to improve the presented preliminary results.

\subsection{Carlova}

Dotto et al. (1995) used the EA method and data from three oppositions $(1979,1984,1986)$ and obtained a model of this asteroid (see Table 3). We were able to calculate the model of Carlova using the lightcurves from six apparitions: 1979, 1984, 1986, 1996, 1997, 1998 (ecliptic latitude from $-15^{\circ}$ to $+16^{\circ}$ ). The results are presented in Table 3 . 
We determined the sidereal period and the prograde rotation of this asteroid. The coordinates of the north pole are close to the first solution of Dotto et al. (1995) and $a / b$ is a little smaller than that obtained by these authors. The difference in the ratios $b / c$ obtained by Dotto et al. (1995) and in the present study is much greater. Dotto et al. (1995) used only the EA method and the A approch is not so good for $b / c$ determination. The magnitude (M) method is a much better indicator for $b / c$ and such a method has also been used in the present work (see Michałowski 1993 for details).

\subsection{Vaticana}

There is no previously published model for this asteroid. We have lightcurves from 1985, 1989, 1995-96, and 1998. The unique value of the sidereal period and prograde sense of rotation have been obtained (see Table 3). The ecliptic latitudes of Vaticana during these four apparitions varied from $-21^{\circ}$ to $+18^{\circ}$ but the two-fold ambiguity still exists (see table). It is probably due to the small number of observed oppositions and the future observations should help to resolve this ambiguity.

\subsection{Amherstia}

Using the lightcurves from three oppositions (1978, 1985, 1989), De Angelis (1995) and Michałowski (1996) obtained slightly different parameters, especially for the triaxial ellipsoid model (see Table 3). This problem was analyzed earlier by Michałowski (1996).

The data from five oppositions (1978, 1985, 1989, 1995, 1996) allowed us to determine a model of this asteroid (Table 3). Unfortunately, we have obtained two similar solutions for both prograde and retrograde senses of rotation (the ecliptic coordinates for these solutions indicate two poles of the same axis of rotation). It probably means that Amherstia has a spin vector located in the ecliptic plane. For such asteroids the sense of rotation is undefined.

\subsection{Neckar}

For this asteroid, the data obtained during seven apparitions $(1977,1983,1987,1989,1990,1993,1995-96)$ are available. The model is given in Table 3. As the ecliptic latitude of Neckar is always close to zero (from $-3^{\circ}$ to $+4^{\circ}$ during previous oppositions), two solutions for the north pole have been obtained. The available lightcurves have allowed us to obtain the unique value for the sidereal period and the prograde sense of rotation. The results presented here are the first ones published for 1223 Neckar.

Acknowledgements. This work was partially supported by the Polish KBN Grants 2 P03D 02409 and 2 P03D 00718.

\section{References}

Binzel R.P., 1987, Icarus 72, 135

Bowell E., Hapke B., Domingue D., et al., 1989, in Asteroids II, 524. Univ. of Arizona Press, Tucson

De Angelis G., 1995, Planet. Space Sci. 43, 649

Debehogne H., De Sanctis G., Zappala V., 1983, Icarus 55, 236

Denchev P., Magnusson P., Donchev Z., 1998, Planet. Space Sci. 46,673

Di Martino M., Zappala V., De Campos J.A., et al., 1987, A\&AS 67,95

Dotto E., Barucci M.A., Fulchignoni M., et al., 1992, A\&AS 95, 195

Dotto E., De Angelis G., Di Martino M., et al., 1995, Icarus 117,313

Erikson A., Cutispoto G., Debehogne H., et al., 1991, A\&AS 91, 259

Harris A.W., 1989, In Lunar and Planetary Science XX, 375, Lunar and Planetary Institute, Houston

Harris A.W., Young J.W., 1980, Icarus 43, 20

Harris A.W., Young J.W., 1983, Icarus 54, 59

Kryszczyńska A., Colas F., Berthier J., Michałowski T., Pych W., 1996, Icarus 124, 134

Lagerkvist C.-I., Hahn G., Magnusson P., Rickman H., 1987, A\&AS 70, 21

Magnusson P., Lagerkvist C.-I., 1990, A\&AS 86, 45

Magnusson P., Lagerkvist C.-I., 1991, A\&AS 87, 269

Magnusson P., Barucci M.A., Drummond J.D., et al., 1989, in Asteroids II, 66. Univ. of Arizona Press, Tucson

Michałowski T., 1993, Icarus 106, 563

Michałowski T., 1996, Icarus 123, 456

Michałowski T., Velichko F.P., Di Martino M., et al., 1995, Icarus 118, 292

Miles R., 1990, Minor Planet Bull. 17, 25

Schober H.J., Erikson A., Hahn G., et al., 1994, A\&AS 105, 281

Slivan S.M., Binzel R.P., 1996, Icarus 124, 452

Tedesco E.F., 1979, Ph.D. Thesis, New Mexico State University

Tholen D.J., 1989, In Asteroids II, 1139. Univ. of Arizona Press, Tucson

Udalski A., Pych W., 1992, Acta Astron. 42, 285

Weidenschilling S.J., Chapman C.R., Davis D.R., et al., 1990, Icarus 86, 402

Zappala V., Di Martino M., Cellino A., et al., 1989, Icarus 82, 354

Zappala V., Cellino A., Barucci M.A., et al., 1990, A\&A 231, 548 